\title{
Literature Review of Mental Health and Psychosocial Aspects of Ebola Virus Disease
}

\author{
Anna Grigoryan*1, Rebecca Bitsko², Ha Young Lee ${ }^{3}$, Barbara Lopes-Cardozo ${ }^{3}$ and Ruth \\ Perou $^{2}$
}

${ }^{1}$ National Center for Immunization and Respiratory Diseases, Centers for Disease Control and Prevention (CDC), Atlanta, GA, USA; ${ }^{2}$ National Center on Birth Defects \& Developmental Disabilities, CDC, Atlanta, GA, USA; ${ }^{3}$ Center for Global Health, CDC, Atlanta, GA, USA

\section{Objective}

To present the summary results of a literature review pertinent to mental health and psychosocial aspects of Ebola virus disease (EVD).

\section{Introduction}

The 2014 outbreak of EVD is the largest and most complex Ebola outbreak since 1976 affecting several countries in West Africa. The mental health and psychosocial implications of the 2014 Ebola outbreak are serious and multifaceted, impacting survivors, families, communities, healthcare providers, and the public health response. In addition, psychosocial support is a key priority to the Ebola response. CDC's Ebola Mental Health Team (EMHT) was activated in September 2014. This study has been conducted to support the CDC's EMHT tasks.

\section{Methods}

We searched on-line CDC libraries and public websites for "mental health and Ebola" and "psychosocial and Ebola" word combinations. The scope of the search was expanded to include press releases and newspaper articles due to the urgent nature of the 2014 Ebola outbreak. This review was conducted during November 2014-February 2015 and was limited to information published in English. Particular emphasis was placed on the mental health and psychosocial issues of EVD that might provide further guidance to health care personnel and Ebola responders.

\section{Results}

A total of 140 documents were identified, including peer-reviewed research, newspaper articles, pamphlets, and guidelines/training manuals. As of March 1, 2015 the review of different library resources and websites showed that there are three broad categories of mental health issues: 1) Ebola patients; 2) healthcare providers and Ebola responders; 3) Ebola survivors.

One of the main obstacles in reducing the outbreak has been the widespread ignorance, and potential panic over EVD, leading to fear, isolation, and stigmatization. Using results of this review the CDC's EMHT developed stigma mitigation related messaging, which addressed psychosocial support and stress management for Ebola survivors and responders. Results of the study can help readers to compare identified mental health and psychosocial aspects of Ebola outbreaks to other severe epidemics in order to plan on important public health issues.

\section{Conclusions}

The results of this literature review can be used by healthcare personnel and public health professionals to complement CDC's other guidance documents on EVD. This review could assist with further studies and publications, and facilitate intergovernmental collaboration in the areas of Ebola mental health and psychosocial support and public health practice.
Disclaimer: The views expressed are those of the authors and should not be construed to represent the positions of the Centers for Disease Control and Prevention.

\section{Keywords}

Ebola Virus Disease (EVD); Mental Health; Psychosocial support; Literature Review; Population Health

\section{References}

1. "A time of fear": local, national, and international responses to a large Ebola outbreak in Uganda. Kinsman Globalization and Health 2012, 8:15 2. Ebola: epidemic echoes and the chronicle of a tragedy foretold. Mark Honigsbaum, The Lancet, Volume 384, No 9956, p1740-41, 15 Nov 2014

\section{*Anna Grigoryan}

E-mail: Ffg7@cdc.gov 